\title{
Matter Wave Interferometry, the Gouy Phase and Complementarity Principle
}

\author{
Luis A. Cabral, Irismar G. da Paz, \\ José G. G. de Oliveira Júnior, Saulo Moreira, \\ Marcos D. R. Sampaio and Maria C. Nemes \\ Additional information is available at the end of the chapter \\ http://dx.doi.org/10.5772/53651
}

\section{Introduction}

Over a hundred years ago L. G. Gouy described an anomalous behavior of the phase of a converging diffracted spherical wave as it passes through a focus [1, 2]. He also performed the experiment which confirmed his theoretical analysis. He considered a point source and let it impinge onto two mirrors with different curvature radius, one concave of other plane, parallel to each other. The observational plane was transverse to the so generated beams. Their interference yielded circular interference fringes. Since then many observations of such phase anomaly have been reported, some of them in [3-12].

It turns out that the Gouy phase has found several important applications. As one example we quote the influence of this anomalous phase as being of critical importance for any application as ultrashort laser pulses, including high-harmonics and attosecond pulse generation [13]. It also has important consequences in the optical range of the electromagnetic spectrum such as phase matching in high order harmonic generation governed by atomic response and the Gouy phase of the fundamental radiation [14-16]. On the theoretical side numerous authors have treated the problem an explanation for such phase. One of the first authors to consider the question was P. Debye in 1909, who found an exact solution to the wave equation for all space treating the boundary condition in an elegant way, avoiding its mathematical complication [17]. The anomalous propagation through the focus has been treated by several authors since then. In refs [18, 19] one also finds early rather involved theories explaining the phase anomaly.

Interesting enough even nowadays several authors still consider the Gouy phase as "the most poorly understood subjects in physical optics" [20], although several simple explanation have been offered in terms of Berry geometrical phase [4], the uncertainty principle [10] and so one [5, 6, 21-25].

A successful description of optical waves crossing an aperture is the so called paraxial approximation to the Helmholtz equation (explained below). A physically appealing aspect 
of this equation is its complete correspondence to Schrödinger equation for matter waves. However in the latter case the corresponding phase is a time dependent overall phase and therefore omitted in quantum mechanics text books. In spite of that it is manifest and in fact the agent responsible for the growth of the width gaussian wave packet under a free evolution. One of the objectives of the present contribution is to unveil the similarities between these to apparently independent subjects, which, however bear a strong conceptual basis, which has been recently show $[9,10]$. Also an experimental proposal was put forth for the observation of the Gouy phase of matter waves in the optical regime using Ramsey interferometry with Rydberg atoms [12].

In fact the basis for the interpretation of the anomalous phase of matter waves is the Schrödinger Robertson uncertainty relation which can be immediately carried over for laser light. As will be shown here the Gouy phase in both cases is related to the $x-p$ cross correlations, i.e., the expectation value of the position-symmetrized-momentum. It becomes thus clear that the phase is intimately connected to the correlations generated by the uncertainty relation.

Our second purpose, which is a rather novel investigation relates to the role of the Gouy phase in interference phenomena. Here in particular we restrict ourselves to two slit experiments which, again, are equally both for light or matter.

The first theoretical to be faced is the bridge between the complementarity principle as usually constructed using q-bits and their equivalent for continuous systems. We define and explore the constructed definition for visibility and predictability, showing, in particular that the Gouy phase will be manifest in the number of fringes of a given arrangement. Thus by changing the slit aperture of the grating and measuring the corresponding change in the number of fringes on the screen gives an indirect evidence of this phase. Again the idea that the Gouy phase is at the root of this phenomena is not exclusive of matter or light waves, since it has only to do with wave nature.

In section 2 we make the analogy between the paraxial wave equation for light and the Schrödinger's equation for a free particle. In section 3 we discuss the generalized complementarity principle within a double slit experiment. We quantify the wave-particle duality through the definition of quantities called visibility and predictability which characterize the wave and particle behavior respectively [26, 32]. Section 4 is reserved to a wave quantitative analogies of interferometry of matter waves in double slit experiment showing that the Gouy phase is related to number of fringes. Moreover we show that the Gouy phase can be indirectly extracted by analyzing the change in the interference fringes with the variation of the slit width or the distance of the detector. The results that we will obtain here for matter waves can be equally applied for classical and quantum electromagnetic waves in the paraxial regime. In section 5 we make our conclusions.

\section{From Maxwell to matter: Waves the Gouy phase}

We proceed to exploring the analogies between properties of electromagnetic waves in the paraxial approximation and matter waves. In Fig. 1 we compare plane, spherical and Gaussian wavefronts. The latter is obtained when a converging spherical wave is obstructed by a circular aperture. Notice that a Gaussian wavefront acquires an anomalous phase shift as it passes through the focal region, i.e., their wavefront are slighted advanced in relation to the others. This phase shift is the Gouy phase. 

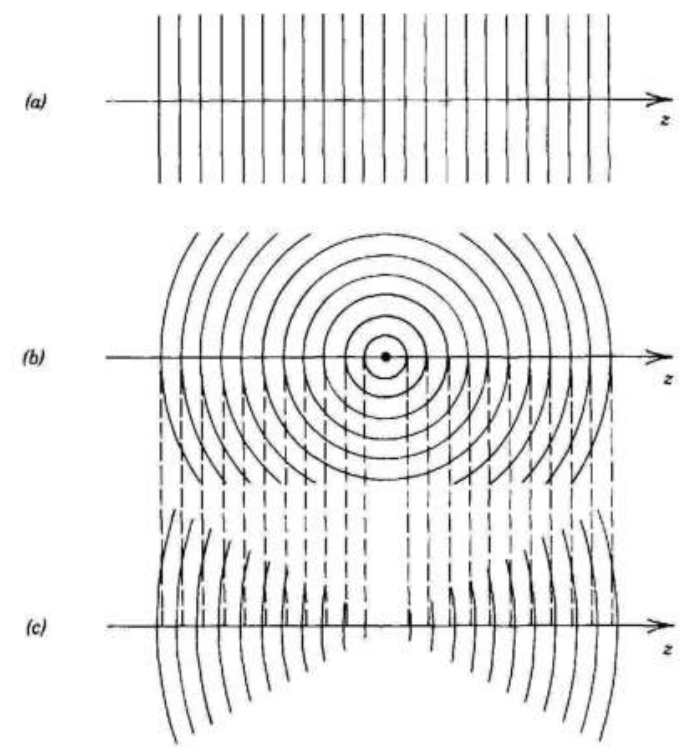

Figure 1. Wavefronts: a) plane wave, b) spherical wave, c) Gaussian beam. Extracted from [ref. B. E. A. Saleh and M. Teich, Fundamentals of Photonics (John Wiley Sons, New York, 1991).]

Consider a stationary electric field in vacuum

$$
E(\vec{r})=A(\vec{r}) \exp (i k z)
$$

where $k$ is the wave number. In the paraxial approximation we assume that the modulus of the wave vector $|\vec{k}| \approx k_{z}$, i.e., $k_{z} \gg k_{x}, k_{y}$. This amounts to saying that the function $A(\vec{r})$ which modulates the field varies slowly compared with the wavelength $\lambda=2 \pi / k$. Under this condition the Helmholtz equation for the electric field is [25]

$$
\left(\frac{\partial^{2}}{\partial x^{2}}+\frac{\partial^{2}}{\partial y^{2}}+i 4 \pi \frac{1}{\lambda_{L}} \frac{\partial}{\partial z}\right) A(x, y, z)=0
$$

where $\lambda_{L}$ is the light wavelength.

On the other hand the two-dimensional Schrödinger equation for a free particle of mass $m$ reads

$$
\left(\frac{\partial^{2}}{\partial x^{2}}+\frac{\partial^{2}}{\partial y^{2}}+2 i \frac{m}{\hbar} \frac{\partial}{\partial t}\right) \psi(x, y, t)=0
$$

Notice that snapshots of $\psi\left(x, y, t_{i}\right)$ is identical to slices for fixed $z_{i}$ of $A\left(x, y, z_{i}\right)$. Therefore we can interpret the time evolution of $\psi(x, y, t)$ in terms of parameter $z$ related to time as $t=z / v_{z}$, where $v_{z}$ can be interpreted in terms of the de Broglie wavelength $\lambda_{P}=h / m v_{z}$ as we assume $p \sim p_{z}$. 
Based on this discussion Equation (3) can formally written as [7-9]

$$
\left(\frac{\partial^{2}}{\partial x^{2}}+\frac{\partial^{2}}{\partial y^{2}}+i 4 \pi \frac{1}{\lambda_{P}} \frac{\partial}{\partial z}\right) \psi\left(x, y, t=z / v_{z}\right)=0
$$

Next we show that the Gouy phase is intimately connected to the generalized Schrödinger-Robertson uncertainty relation [7-10] which provides for a physically appealing interpretation of this phase which can be carried over to light waves in paraxial approximation.

The solution of Equation (2) for a transverse Gaussian beam collimated in the $z$ direction is

$$
A_{G}(x, z)=A_{0} \frac{w_{0}}{w(z)} \exp \left[-\frac{x^{2}}{w(z)^{2}}+i \frac{k x^{2}}{2 R(z)}+i \xi(z)\right]
$$

where we dropped the $y$ component for brevity. In the equation above, $w(z)$ is the beam width, $R(z)$ is the curvature radius, $\xi(z)$ is the Gouy phase and $z_{0}$ is the Rayleigh range given by

$$
\begin{gathered}
w(z)=w_{0}\left[1+\left(\frac{z}{z_{0}}\right)^{2}\right]^{\frac{1}{2}}, \quad R(z)=z\left[1+\left(\frac{z_{0}}{z}\right)^{2}\right], \\
\xi(z)=-\frac{1}{2} \arctan \left(\frac{z}{z_{0}}\right), \quad z_{0}=\frac{k w_{0}^{2}}{2} .
\end{gathered}
$$

The total variation of the Gouy phase as we go from $z=-\infty$ to $z=+\infty$ is $\pi / 2$ as we can immediately verify from (7). This change is abrupt and effectively takes place in the so called Rayleigh range $z_{0}$. On the other hand, solution of Equation (4) for an initial condition given by a Gaussian wave packet is given by [7-9]

$$
\psi(x, t)=\frac{1}{\sqrt{\sqrt{\pi} B(t)}} \exp \left[-\frac{x^{2}}{2 B(t)^{2}}+i \frac{m x^{2}}{2 \hbar R(t)}+i \mu(t)\right]
$$

From the solutions (5) and (8) we can make the following identifications

$$
\begin{gathered}
w(z) \longrightarrow B(t)=b_{0}\left[1+\left(\frac{t}{\tau_{0}}\right)^{2}\right]^{\frac{1}{2}}, \\
R(z) \longrightarrow R(t)=t\left[1+\left(\frac{\tau_{0}}{t}\right)^{2}\right],
\end{gathered}
$$




$$
\xi(z) \longrightarrow \mu(t)=-\frac{1}{2} \arctan \left(\frac{t}{\tau_{0}}\right)
$$

and

$$
z_{0} \longrightarrow \tau_{0}=\frac{m b_{0}^{2}}{\hbar}
$$

To get a better insight inter the role played by $\tau_{0}$ (or equivalently the Rayleigh range $z_{0}$ ) is convenient to rewrite (8) as

$$
\psi(x, t)=\tilde{\varphi}(x, t) \exp [i S(x, t)]
$$

where

$$
\tilde{\varphi}(x, t)=\frac{1}{\sqrt{\sqrt{\pi} B(t)}} \exp \left[-\frac{x^{2}}{2 B(t)^{2}}\right]
$$

and

$$
S(x, t)=\frac{x^{2}}{2 B(t)^{2}} \frac{t}{\tau_{0}}+\mu(t) .
$$

Notice that the position dependence phase $S$ contains the time scale $\tau_{0}$. the ratio $t / \tau_{0}$ will determine the importance of this $x$-dependent phase to the interference pattern. In the experimental setups using fullerene molecules [36] $t / \tau_{0} \approx 10^{4}$ which is also the condition for Frauhoffer diffraction (see ref. [27]). The time scale $\tau_{0}$ is fundamentally determined by Heisenberg's uncertainty relation, given the initial position dispersion $\Delta x(0)=\sigma_{0} / \sqrt{2}$. In fact, the corresponding momentum dispersion is $\Delta p=\hbar /\left(\sigma_{0} \sqrt{2}\right)$. Because the momentum is a constant of motion this momentum spread will be preserved in time. Both $\Delta x$ and $\Delta p$ constitute intrinsic properties of the initial wave packet, in terms of which the time scale $\tau_{0}$ is expressed as

$$
\tau_{0}=\frac{\Delta x(0)}{(\Delta p) / m}
$$

The numerator in the above relation represents the spatial dimensions of the initial wave packet, whilst the denominator stands for the scale of velocity difference enforced by the uncertainty principle. Therefore the time scale $\tau_{0}$ corresponds essentially to the time during which a distance of the order of the wave packet extension is traversed with a speed corresponding to the dispersion in velocity. It can therefore be viewed as a characteristic time for the "aging" of the initial state, which consists in components with larger velocities (relatively to the group velocity of the wave packet) concentrating at the frontal region of the packet. This can be seen explicitly by deriving the velocity field associated with the phase $S$ in Equation (15), which reads 


$$
v(x, t)=\frac{\hbar}{m} \frac{\partial S}{\partial x}=\frac{t x}{\tau_{0}^{2}+t^{2}} .
$$

This expression shows that for $t>0$ the initial velocity field $v(x, 0)=0$ varies linearly with respect to the distance from center of the wave packet $(x=0)$.

Next we relate quantitatively this "aging" effect to position-momentum correlations. This is readily achieved using the generalized uncertainty relation devised by Schrödinger [28], which is expressed in this case in terms of the determinant of the covariance matrix

$$
\operatorname{det} \sum \equiv \operatorname{det}\left(\begin{array}{cc}
\sigma_{x x} & \sigma_{x p} \\
\sigma_{x p} & \sigma_{p p}
\end{array}\right) \geq \frac{\hbar^{2}}{4}
$$

For the minimum uncertainty wave packet of Equation (8) we obtain, at all times,

$$
\operatorname{det}\left(\begin{array}{ll}
\sigma_{x x} & \sigma_{x p} \\
\sigma_{x p} & \sigma_{p p}
\end{array}\right)=\frac{\hbar^{2}}{4}
$$

Therefore we establish a direct relation between the Gouy phase and the position-momentum correlation for matter waves,

$$
\sigma_{x p}=\frac{\hbar t}{2 \tau_{0}}=-\frac{\hbar}{2} \tan 2 \mu(t) .
$$

For light waves a similar interpretation applies,

$$
\sigma_{x k_{x}}=\frac{z}{2 z_{0}}=-\frac{1}{2} \tan 2 \zeta(z)
$$

The formulation of the generalized uncertainty relation for light waves in terms of operators can be found in [29]

\section{The complementarity principle in double slit experiment}

Wave-particle duality as first conceived by [30] stated that in a given experimental set up one observes either wave or particle properties. Using his words: "we are presented with a choice of either tracing the path of a particle or observing interference effects". In double slit experiments the wave nature of the object is reflected by the interference pattern exhibited on a screen. We know however that once the knowledge of the object's path (i.e., through which slit it crossed) is obtained, the interference pattern is completely destroyed. A natural question that can arise is how partial knowledge of path affects such pattern. This discussion was first addressed by [31] and later by [32]. The quantification of which-way information 
(predictability) and visibility was established by [33] and the relation between both given by [34].

We proceed to define visibility and predictability in two slit experiments. Consider a double slit experiment as depicted in the Fig. 2. $B$ and $C$ denote the slits, $A$ the position from which the particles are emitted and $D$ the detector which covers the whole extension of the screen and counts the numbers of particles. Notice that $A$ need not be placed on the symmetry axis $O x$.

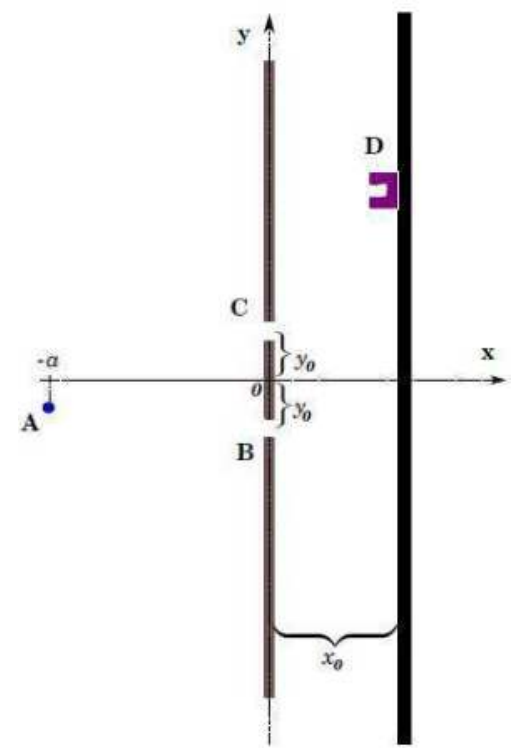

Figure 2. Illustration of the double slit with a displaced source of particles.

Suppose $N$ particles are sent to the grating. Consider that $\left(N_{B}\right) N_{C}$ particles cross slit $(B) C$ leaving slit $(C) B$ closed with relative probability $\left(P_{B}\right) P_{C}$.

The probability amplitude at the screen is

$$
\psi\left(x_{0}, y\right)=\sqrt{P_{B}} \psi_{B}\left(x_{0}, y\right)+\sqrt{P_{C}} \psi_{C}\left(x_{0}, y\right)
$$

The probability density of finding a particle at the point $\left(x_{0}, y\right)$ on the screen knowing that it crossed slit $B$ is obviously

$$
\mathrm{P}_{B}(y)=P_{B}\left|\psi_{B}\left(x_{0}, y\right)\right|^{2}
$$

Analogously

$$
P_{C}(y)=P_{C}\left|\psi_{C}\left(x_{0}, y\right)\right|^{2},
$$


for slit $C$.

Now the quantification of which way information, also known as predictability is

$$
\mathcal{P}(y)=\left|\frac{P_{B}(y)-P_{C}(y)}{P_{B}(y)+P_{C}(y)}\right| .
$$

The visibility $\mathcal{V}$ is defined through the expression for the intensity $\left|\psi\left(x_{0}, y\right)\right|^{2}$ on the screen

$$
I(y)=P_{B}\left|\psi_{B}\left(x_{0}, y\right)\right|^{2}+P_{C}\left|\psi_{C}\left(x_{0}, y\right)\right|^{2}+2\left|\sqrt{P_{B} P_{C}} \psi_{B}\left(x_{0}, y\right) \psi_{C}\left(x_{0}, y\right)\right| \cos \phi,
$$

with $\phi=\arg \psi_{B}\left(x_{0}, y\right)-\arg \psi_{C}\left(x_{0}, y\right)$.

We can rewrite $I(y)$ as

$$
I(y)=\left[\mathrm{P}_{B}(y)+\mathrm{P}_{C}(y)\right]\left[1+2\left|\frac{\sqrt{P_{B} P_{C}} \psi_{B}\left(x_{0}, y\right) \psi_{C}\left(x_{0}, y\right)}{\mathrm{P}_{B}(y)+\mathrm{P}_{C}(y)}\right| \cos \phi\right],
$$

from which the visibility [37]

$$
\mathcal{V}=\frac{I_{\max }-I_{\min }}{I_{\max }+I_{\min }}
$$

can be read off as

$$
\mathcal{V}=\frac{2\left|\sqrt{P_{B} P_{C}} \psi_{B}\left(x_{0}, y\right) \psi_{C}\left(x_{0}, y\right)\right|}{P_{B}(y)+P_{C}(y)}
$$

We can immediately verify that $[32,34]$

$$
\mathcal{P}^{2}+\mathcal{V}^{2}=1
$$

for all $y$. The relation above is known as the complementarity relation.

\section{Double slit interference: Complementarity principle and the Gouy phase}

In this section we discuss double-slit interference of matter waves, for instance neutrons or fullerene molecules [35], to illustrate the ideas that we discussed so far. Consider the experimental set up illustrated in the Fig. 3. $b_{0}$ is the characteristic width of a wavepacket, assumed to be coherent, i.e., not affected by indeterminacies inherent to the production process, $\sigma_{0}$ is the slit widths, $x_{0}$ the distance between the slit grating and screen and $d$ is the distance between the slits. The particles travel in the $x$ direction with speed $v$, assuming that the center of mass moves classically, that is $\Delta p_{x} \ll p_{x}$.

The intensity on the screen reads 


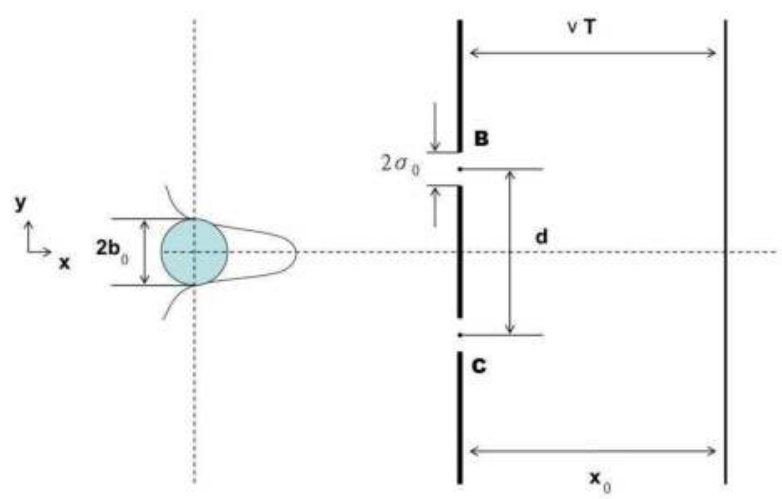

Figure 3. Experimental set up.

$$
I\left(y, T=x_{0} / v\right)=\left|\sqrt{P_{B}} \psi_{B}\left(y, T=x_{0} / v\right)+\sqrt{P_{C}} \psi_{C}\left(y, T=x_{0} / v\right)\right|^{2}
$$

where

$$
\begin{aligned}
& \psi_{B}(y, T)=\frac{1}{\sqrt{B(T) \sqrt{\pi}}} \exp \left[-\frac{\left(y+\frac{d}{2}\right)^{2}\left(1-i \frac{T}{\tau_{0}}\right)}{2 B(T)^{2}}+i \mu(T)\right], \\
& \psi_{C}(y, T)=\frac{1}{\sqrt{B(T) \sqrt{\pi}}} \exp \left[-\frac{\left(y-\frac{d}{2}\right)^{2}\left(1-i \frac{T}{\tau_{0}}\right)}{2 B(T)^{2}}+i \mu(T)\right],
\end{aligned}
$$

and $\tau_{0}=\frac{m \sigma_{0}^{2}}{\hbar}$. An analytical expression for $\mathrm{P}_{B}$ and $\mathrm{P}_{C}$ can in principle be derived in terms of the parameters as illustrated in Fig. 3 as performed in [35] but it is not essential to the purposes of our discussion.

It is easy to show that $I(y, T)$ can be written as

$$
I(y)=F(y)\left[1+\frac{2 \sqrt{P_{B} P_{C}}}{P_{B} e^{\alpha y}+P_{C} e^{-\alpha y}} \cos (\beta y)\right],
$$

where 


$$
\begin{gathered}
F(y)=I_{0} e^{-\gamma(y)} \frac{P_{B} e^{\alpha y}+P_{C} e^{-\alpha y}}{2 \sqrt{P_{B} P_{C}}}, \\
\gamma(y)=\frac{y^{2}+\left(\frac{d}{2}\right)^{2}}{B(T)^{2}} \\
\alpha=\frac{d}{B(T)^{2}}=\frac{1}{\sigma_{x x}} \frac{d}{2}
\end{gathered}
$$

and

$$
\beta=\frac{T}{\tau_{0}} \frac{d}{B(T)^{2}}=\frac{\sigma_{x p}}{\sigma_{x x}} \frac{d}{\hbar}
$$

where hereafter we write $I(y, T)=I(y)$ for sake of brevity.

Recalling the expression for predictability and visibility (25), (29) we get

$$
\begin{gathered}
\mathcal{V}(y)=\frac{2 \sqrt{P_{B} P_{C}}}{P_{B} e^{\alpha x}+P_{C} e^{-\alpha y}}, \\
\mathcal{P}(y)=\left|\frac{P_{B} e^{\alpha y}-P_{C} e^{-\alpha y}}{P_{B} e^{\alpha y}+P_{C} e^{-\alpha y}}\right|,
\end{gathered}
$$

and

$$
\mathcal{P}^{2}(y)+\mathcal{V}^{2}(y)=1
$$

Let $p=\frac{P_{C}}{P_{B}}$. From expression (39) we can find the point on the screen where the visibility reaches its maximal value,

$$
y_{\max }=\frac{\ln \sqrt{p}}{\alpha} .
$$

At this point the which way information is minimum, that is to say $\mathcal{P}\left(y_{\max }\right)=0$ and $\mathcal{V}\left(y_{\max }\right)=1$. Notice that it. In Fig. 4 we illustrate this with $p=1,0.15$ and 0.015 .

For $p=1$, illustrated in Fig. 4, we have 

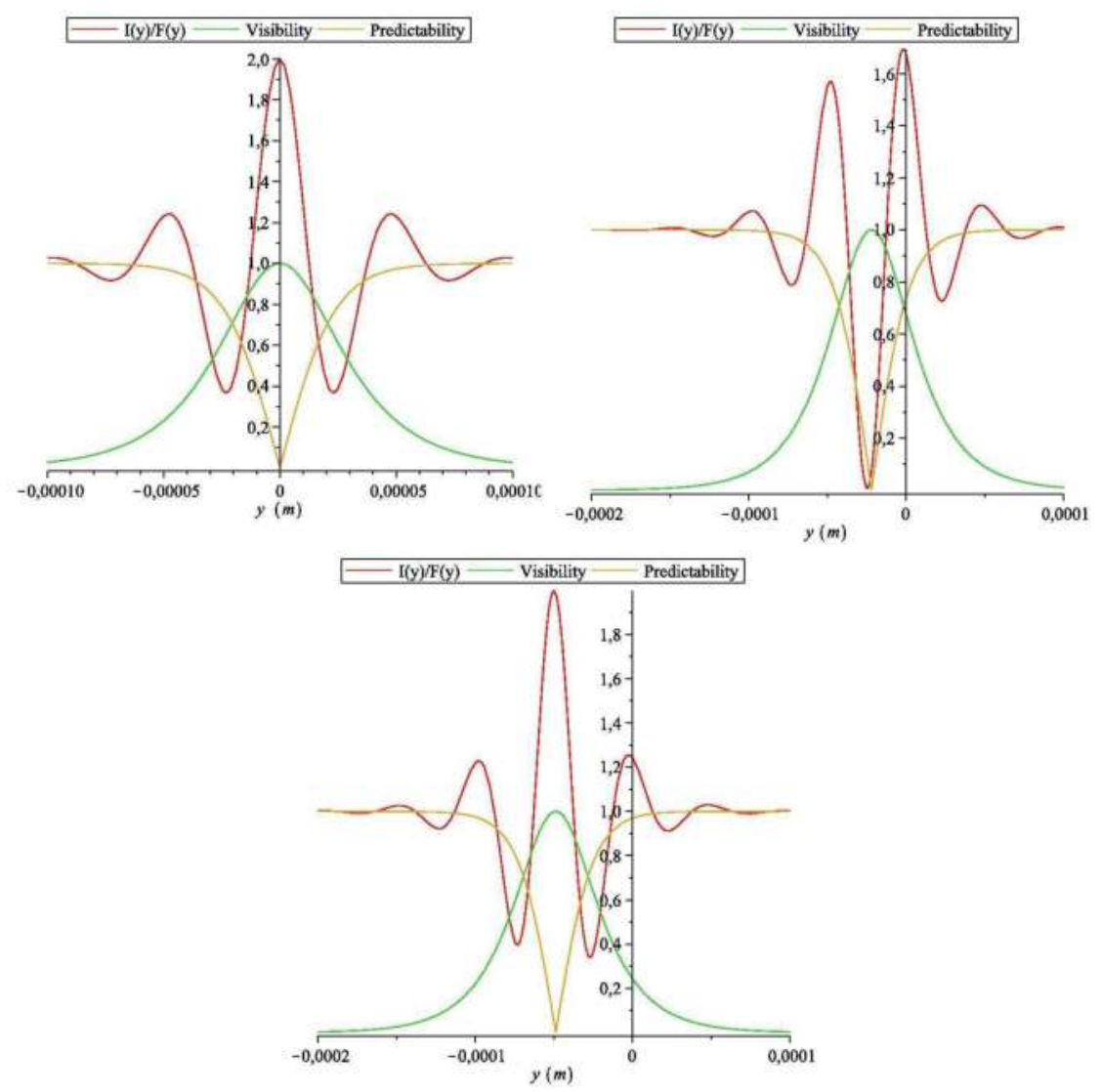

Figure 4. Intensity, visibility and predictability for $p=1, p=0.15$ and $p=0.015$, respectively.

$$
\begin{gathered}
I(y)=F(y)\left[1+\frac{\cos (\beta y)}{\cosh (\alpha y)}\right] \\
\mathcal{V}(y)=\frac{1}{\cosh (\alpha y)}
\end{gathered}
$$

and

$$
\mathcal{P}(y)=|\tanh (\alpha y)|
$$

The peaks of intensity and visibility occurs at $y=0$ where predictability is minimum. In terms of the complementarity principle we have the minimum of knowledge about from 
which slit the particle cames from. Note also that we have intermediate situations where we have interference fringes with $0<\mathcal{P}<1$ and $0<\mathcal{V}<1$, signalizing the existence of situations where the wave like property does not preclude the particle like property.

An important quantity is the ratio $R$ between $\alpha$ and $\beta$,

$$
R=\frac{\alpha}{\beta}=\frac{\hbar}{2 \sigma_{x p}}
$$

It can be used to estimate the effective number of fringes (contrast) $v$ before the visibility decreases by a factor of $1 / e$ [26]

$$
v=\frac{0.264}{R}
$$

for instance, $R=1$ for $v=0.264$.

It is also related to the Gouy phase. From Equations (20) and (46) we get

$$
\mu(T)=-\frac{1}{2} \arctan \left(\frac{1}{R}\right)
$$

In Fig. 5 we plot $\mu$ against the slit width $\sigma_{0}$. We immediately verify that for $\sigma_{0}$ between 0 and $0.02 \mu \mathrm{m}$ the phase varies abruptly from -0.05 to $\frac{\pi}{4}$ rad. For values of the Gouy phase within this range we associated a noticeable contrast in the intensity curves as shown in Fig. 5. When the Gouy phase tends to zero the interference fringes tends to disappear because the geometrical optical regime is attained.
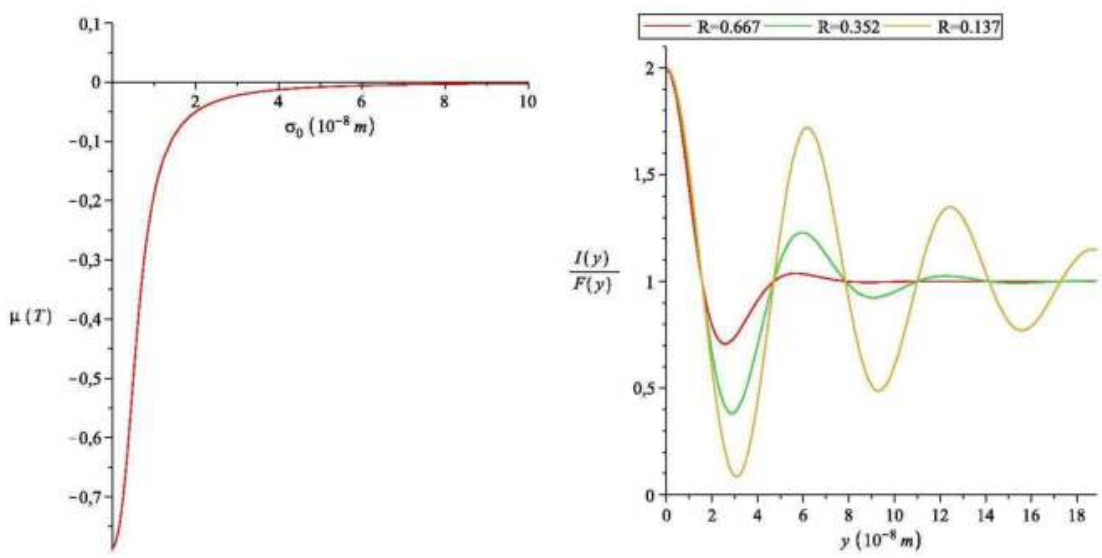

Figure 5. Gouy phase and intensity contrast according Gouy phase. 


\section{Conclusion}

Our results show how partial knowledge of path affects the interference pattern. The position of the peaks of intensity and visibility are affected by the probabilities of the particles cross the slit $B\left(P_{B}\right)$ or $C\left(P_{C}\right)$. When $P_{B}=P_{C}$ these peaks are centered in the position $y_{\max }=0$ and when $P_{B} \neq P_{C}$ they are displaced from this position. In positions $y \neq y_{\text {max }}$ we have the situations where $\mathcal{V}$ and $\mathcal{P}$ are both different of zero, i.e., the wave like property not exclude the particle like property. The crucial point here is the connection between the complementarity principle and the Gouy phase of matter waves. We see that this phase appears in the number of interference fringes exhibiting the wave behavior of the matter. We see that the number of fringes tends to disappear when the Gouy phase tends to zero.

\section{Acknowledgments}

This work was in part supported by the Brazilian agencies $\mathrm{CNPq}$, Capes, Fapepi and Fapemig.

\section{Author details}

Luis A. Cabral ${ }^{1}$, Irismar G. da Paz², José G. G. de Oliveira Júnior ${ }^{3}$, Saulo Moreira ${ }^{4}$, Marcos D. R. Sampaio ${ }^{4}$ and Maria C. Nemes ${ }^{4}$

1 Departamento de Física, Universidade Federal do Tocantins, Brazil

2 Departamento de Física, Universidade Federal do Piauí, Brazil

3 Departamento de Ciências Exatas e Tecnológicas, Universidade Estadual de Santa Cruz, Brazil

4 Departamento de Física, Universidade Federal de Minas Gerais, Brazil

\section{References}

[1] Gouy, L. G. (1890). Sur une propriete nouvelle des ondes lumineuses, Acad. Sci. Paris $110,1251$.

[2] Gouy, L. G. (1891). Sur la propagation anomale des ondes, Ann. Chim. Phip. Ser. 6 24, 145.

[3] Berry, M. V. (1984). Quantal phase factors accompanying adiabatic changes, Proc. R. Soc. London A 392, 45.

[4] Simon, R., and Mukunda, N. (1993). Bargman Invariant and the Geometry of the Gouy Effect, Phys. Rev. Lett. 70, 880.

[5] Feng, S., and Winful, H. G. (2001). Physical origin of the Gouy phase shift, Opt. Lett. 26,485 .

[6] Yang, J., and Winful, H. G. (2006) Generalized eikonal treatment of the Gouy phase shift, Opt. Lett. 31, 104. 
[7] da Paz, I. G. (2006). Matter Waves and Paraxial Propagation of Light, Department of Physics, UFMG, MG, Brazil.

[8] da Paz, I. G. (2011). Gouy phase in matter waves: from pure and mixed Gaussian states, Department of Physics, UFMG, MG, Brazil.

[9] da Paz, I. G., Nemes, M. C., and Peixoto de Faria, J. G. (2011). Electromagnetic Waves Chapter four - Edited by Vitaliy Zhurbenko, InTech, Janeza Trdine 9, 51000 Rijeka, Croatia.

[10] da Paz, I. G., Monken, C. H., Padua, S., Nemes, M. C., and Peixoto de Faria, J. G. (2010). Indirect evidence for the Gouy phase for matter waves, Phys. Lett. A 374, 1660.

[11] da Paz, I. G., Nemes, M. C., and Peixoto de Faria, J. G. (2007). Gouy phase and matter waves, J. Phys.: Conference Series 84, 012016.

[12] da Paz, I. G., Saldanha, P. L., Nemes, M. C., and Peixoto de Faria, J. G. (2011). Experimental proposal for measuring the Gouy phase of matter waves, New Journal of Physics 13, 125005.

[13] Lindner, F., Paulus, G. G., Walther, H., Batuska, A., Goulielmakis, E., Lezius, M., and Krausz, F. (2004). Gouy Phase Shift for Few-Cycle Laser Pulses, Phys. Rev. Lett. 92, 113001.

[14] Balcou, P., and LŠHuillier, A. (1993). Phase-matching effects in strong-field harmonic generation, Phys. Rev. A 47, 1447.

[15] Lewenstein, M., Salières, P., and LŠHuillier, A. (1995). Phase of the atomic polarization in high-order harmonic generation, Phys. Rev. A 52, 4747.

[16] Lindner, F., Stremme, W., Schätzel, M. G., Grasbon, F., Paulus, G. G., Walther, H., Hartmann, R., and Strüder, L. (2003). High-order harmonic generation at a repetition rate of $100 \mathrm{kHz}$, Phys. Rev. A 68, 013814.

[17] Debye, P. (1905). Ann. Phys. 30, 755.

[18] Rubinowicz, A. (1938). On the Anomalous Propagation of Phase in the Focus, Phys. Rev. 54, 931.

[19] Linfoot, E. H. (1956). Proc. Phys. Soc. London 69, 823.

[20] Visser, T. D., and Wolf, E. (2010). The origin of the Gouy phase anomaly and its generalization to astigmatic wavefields, Opt. Comm. 283, 3371.

[21] Boyd, R. W. (1980). Intuitive explanation of the phase anomaly of focused light beams, J. Opt. Soc. Am. 70, 877.

[22] Hariharan, P., and Robinson, P. A. (1996). The Gouy phase shift as a geometrical quantum effect, J. Mod. Opt. 43, 219.

[23] Born, M., and Wolf, E. (1999). Principles of Optics, Cambridge University Press, Cambridge. 
[24] Mandel, L., and Wolf, E. (1995). Optical Coherence and Quantum Optics, Cambridge, New York.

[25] Saleh, B. E. A., and Teich, M. C. (1991). Fundamentals of Photonics, John Wiley et Sons, New York.

[26] Bramon, A., Garbarino.,and Hiesmayr, B. C. (2004). Quantitative complementarity in two-path interferometry, Phys. Rev. A 69, 022112.

[27] Viale, A., Vicari, M., and Zanghì, N. (2003). Analysis of the loss of coherence in interferometry with macromolecules, Phys. Rev. A 68, 063610.

[28] Schrödinger, E. (1930). Zum Heisenbergschen Unsch"arfeprinzip,Proceedings of the Prussian Academy of Sciences XIX pp. 296 Ü303.

[29] Stoler, D. (1981). Operator methods in physical optics, J. Opt. Soc. Am. 71, 334.

[30] Bohr, N. (1928). The quantum postulate and the recent development of atomic theory, Nature 121, 580.

[31] Wootters, W. K., and Zurek, W. K. (1979). Complementarity in the double-slit experiment: Quantum nonseparability and a quantitative statement of Bohr's principle, Phys. Rev. D 19, 473.

[32] Greenberger, D. M., and Yasin, A. (1988). Simultaneous wave and particle knowledge in a neutron interferometer, Phys. Lett. A 128, 391.

[33] Mandel, L. (1991). Coherence and indistinguishability, Opt. Lett. 16, 1882.

[34] Englert, B. G. (1996). Fringe Visibility and Which-Way Information: An Inequality, Phys. Rev. Lett. 77, 2154.

[35] Glionna, G., Blin, A. H., Nemes, M. C., Sampaio, M., and Toledo Piza, A. F. R. (2008). Dispersion and uncertainty in multislit matter wave diffraction, Physica A 387, 1485.

[36] Nairz, O., Arndt, M., and Zeilinger, A. (2002). Experimental verification of the Heisenberg uncertainty principle for fullerene molecules, Phys. Rev. A 65, 032109.

[37] Scully, M. O., and Zubairy, M. S. (1997). Quantum Optics, Cambridge University Press, Cambridge. 
